\title{
A modified Adomian decomposition method for singular initial value Emden-Fowler type equations
}

\author{
J. Biazar ${ }^{1}$, K. Hosseini ${ }^{1,2,3}$ * \\ ${ }^{1}$ Department of Mathematics, Rasht Branch, Islamic Azad University, Rasht, Iran \\ ${ }^{2}$ Department of Mathematics, Guilan Science and Research Branch, Islamic Azad University, Rasht, Iran \\ ${ }^{3}$ Department of Applied Mathematics, Ahrar Institute of Technology and Higher Education, Rasht, Iran \\ *Corresponding author E-mail: kamyar_hosseini@yahoo.com
}

\begin{abstract}
Traditional Adomian decomposition method (ADM) usually fails to solve singular initial value problems of Emden-Fowler type. To overcome this shortcoming, a new and effective modification of ADM that only requires calculation of the first Adomian polynomial is formally proposed in the present paper. Three singular initial value problems of Emden-Fowler type with $\alpha=1,2$, and $>2$, and have been selected to demonstrate the efficiency of the method.
\end{abstract}

Keywords: Singular Initial Value Problems; Emden-Fowler Type; Adomian Decomposition Method; New Modification.

\section{Introduction}

Singular initial value problems play a fundamental role in a wide range of scientific disciplines. In this article, a special type of singular initial value problems which can be expressed as the following form is investigated.

$$
u^{\prime \prime}+\frac{\alpha}{x} u^{\prime}+g(x) h(u)=k(x), \quad u(0)=a, \quad u^{\prime}(0)=b .
$$

Singularity behaviour that occurs at $x=0$ is the main difficulty of this type of initial value problems. In recent years, a variety of methods have been adopted to handle this type of initial value problems. For example, Wazwaz employed a general approach for constructing the exact and series solution of this problem by means of the variational iteration method [1]. Parand et al. applied an approximation algorithm for the solution of this problem using Hermite functions, as basis functions, and collocation method [2]. Parand et al. also adopted a pseudospectral technique based on the rational Legendre functions and Gauss-Radau integration to handle this problem [3]. For further methods, the reader is referred to the references [4-19]. In the present article, ADM is modified effectively to solve presented singular initial value problem. The rest of this article is arranged as follows:

In Section 2, the basic ideas of the method are expressed with details. In Section 3, the proposed method is implemented to solve three singular initial value problems of Emden-Fowler type with $\alpha=1,2$, and $>2$. Finally, Section 4 is devoted to presenting conclusion.

\section{Modified ADM}

Let's consider the following nonlinear equation

$$
u^{\prime \prime}+R(u)+N(u)=f(x)
$$

with the following initial conditions

$\mathrm{u}(0)=\xi_{1}, \quad \mathrm{u}^{\prime}(0)=\xi_{2}$,

where $\mathrm{R}$ is a linear operator, $\mathrm{N}$ is a nonlinear operator, and $\mathrm{f}(\mathrm{x})$ is a known function. It is assumed that the unknown function $u(x)$ can be presented by an infinite series, say

$\mathrm{u}(\mathrm{x})=\sum_{\mathrm{n}=0}^{+\infty} \mathrm{u}_{\mathrm{n}}(\mathrm{x})$

and the nonlinear term $\mathrm{N}(\mathrm{u})$ can be expressed as an infinite series of polynomials given by

$\mathrm{N}(\mathrm{u})=\sum_{\mathrm{n}=0}^{+\infty} \mathrm{A}_{\mathrm{n}}\left(\mathrm{u}_{0}, \mathrm{u}_{1}, \ldots, \mathrm{u}_{\mathrm{n}}\right)$

where $A_{n}, n=0,1, \ldots$ are called the Adomian polynomials and are defined by [20]

$A_{n}=\left\{\begin{array}{lc}N\left(u_{0}\right) & n=0, \\ \frac{1}{n} \sum_{i=0}^{n-1}(i+1) u_{i+1} \frac{d A_{n-1-i}}{d_{0}}, & n=1,2, \ldots .\end{array}\right.$

Applying the inverse operator $\mathrm{L}^{-1}=\int_{0}^{\mathrm{X}} \int_{0}^{\mathrm{X}}(.) \mathrm{dxdx}$, on both sides of Eq. (1) and considering (2) and (3), leads to

$\sum_{n=0}^{+\infty} u_{n}=u(0)+u^{\prime}(0) x+L^{-1}[f(x)]$

$-\mathrm{L}^{-1}\left[\mathrm{R}\left(\sum_{\mathrm{n}=0}^{+\infty} \mathrm{u}_{\mathrm{n}}\right)+\sum_{\mathrm{n}=0}^{+\infty} \mathrm{A}_{\mathrm{n}}\left(\mathrm{u}_{0}, \mathrm{u}_{1}, \ldots, \mathrm{u}_{\mathrm{n}}\right)\right]$. 
We rewrite (4) as follows [21], [22]

$$
\begin{aligned}
& \sum_{n=0}^{+\infty} u_{n}=u(0)+u^{\prime}(0) x \\
& +L^{-1}\left[\sum_{n=0}^{+\infty} a_{n} x^{n}\right]-p L^{-1}\left[\sum_{n=0}^{+\infty} a_{n} x^{n}\right]+L^{-1}[f(x)] \\
& -L^{-1}\left[R\left(\sum_{n=0}^{+\infty} u_{n}\right)+\sum_{n=0}^{+\infty} A_{n}\left(u_{0}, u_{1}, \ldots, u_{n}\right)\right],
\end{aligned}
$$

where $\mathrm{p}$ is an artificial parameter and $\mathrm{a}_{\mathrm{i}}, \mathrm{i}=0,1, \ldots$ are unknown coefficients. We now define

$$
\begin{aligned}
& \mathrm{u}_{0}=\xi_{1}+\xi_{2} \mathrm{x}+\mathrm{L}^{-1}\left[\sum_{\mathrm{n}=0}^{+\infty} \mathrm{a}_{\mathrm{n}} \mathrm{x}^{\mathrm{n}}\right], \\
& \mathrm{u}_{1}=\mathrm{L}^{-1}[\mathrm{f}(\mathrm{x})]-\mathrm{pL}^{-1}\left[\sum_{\mathrm{n}=0}^{+\infty} \mathrm{a}_{\mathrm{n}} \mathrm{x}^{\mathrm{n}}\right]-\mathrm{L}^{-1}\left[\mathrm{R}\left(\mathrm{u}_{0}\right)+\mathrm{A}_{0}\left(\mathrm{u}_{0}\right)\right], \\
& \mathrm{u}_{2}=-\mathrm{L}^{-1}\left[\mathrm{R}\left(\mathrm{u}_{1}\right)+\mathrm{A}_{1}\left(\mathrm{u}_{0}, \mathrm{u}_{1}\right)\right], \\
& \mathrm{u}_{3}=-\mathrm{L}^{-1}\left[\mathrm{R}\left(\mathrm{u}_{2}\right)+\mathrm{A}_{2}\left(\mathrm{u}_{0}, \mathrm{u}_{1}, \mathrm{u}_{2}\right)\right],
\end{aligned}
$$

To avoid calculation of $A_{n}, n=1,2, \ldots$, let determine $a_{i}, i=0,1, \ldots$ such that $\mathrm{u}_{1}=0$. This implies that

$\mathrm{u}_{2}=\mathrm{u}_{3}=\cdots=0$

Setting $\mathrm{p}=1$, yields the solution of Eq. (1) with the initial conditions as follows

$$
u(x)=\xi_{1}+\xi_{2} x+L^{-1}\left[\sum_{n=0}^{+\infty} a_{n} x^{n}\right]
$$

\section{Application}

In this section, three singular initial value Emden-Fowler type equations, including a homogeneous nonlinear Emden-Fowler equation with $\alpha=1$, and two inhomogeneous Emden-Fowler equations, with $\alpha=2$ and $\alpha>2$ will be solved to illustrate the efficiency of the method. The computations associated with these examples have been performed by Maple package.

Example 3.1: Consider the homogeneous nonlinear Emden-Fowler equation with $\alpha=1$ [1]

$u^{\prime \prime}+\frac{1}{x} u^{\prime}-u^{3}+3 u^{5}=0$,

with the following initial conditions

$\mathrm{u}(0)=1, \quad \mathrm{u}^{\prime}(0)=0$.

Traditional ADM. As we know in the traditional ADM, we will reach the following expression

$\sum_{\mathrm{n}=0}^{+\infty} \mathrm{u}_{\mathrm{n}}=\mathrm{u}(0)+\mathrm{u}^{\prime}(0) \mathrm{x}$

$-L^{-1}\left[R\left(\sum_{n=0}^{+\infty} u_{n}\right)+\sum_{n=0}^{+\infty} A_{n}\left(u_{0}, u_{1}, \ldots, u_{n}\right)\right]$,

where $\mathrm{L}^{-1}=\int_{0}^{\mathrm{X}} \int_{0}^{\mathrm{X}}(.) \mathrm{dxdx}, \mathrm{R}(\mathrm{u})=(1 / \mathrm{x}) \mathrm{u}^{\prime}$, and $\mathrm{A}_{\mathrm{n}}, \mathrm{n}=0,1, \ldots$ are as the following

$\mathrm{A}_{0}=-\mathrm{u}_{0}^{3}+3 \mathrm{u}_{0}^{5}$
$A_{1}=-3 u_{0}^{2} u_{1}(x)+15 u_{0}^{4} u_{1}(x)$

We can define

$\mathrm{u}_{0}=\mathrm{u}(0)+\mathrm{u}^{\prime}(0) \mathrm{x}$,

$u_{n}=-L^{-1}\left[R\left(u_{n-1}\right)+A_{n-1}\left(u_{0}, u_{1}, \ldots, u_{n-1}\right)\right], \quad n=1,2, \ldots$.

Therefore

$\mathrm{u}_{0}(\mathrm{x})=1, \quad \mathrm{u}_{1}(\mathrm{x})=-\mathrm{x}^{2}$,

$u_{2}(x)=x^{2}+x^{4}, u_{3}(x)=-x^{2}-\frac{4}{3} x^{4}-\frac{13}{10} x^{6}$,

Now, the series solution derived by the traditional ADM can be written as follows

$u(x)=1-x^{2}-\frac{1}{3} x^{4}-\frac{13}{10} x^{6}+\cdots$

Modified ADM. To solve the problem by the modified ADM, let us rewrite (5) as follows

$\sum_{\mathrm{n}=0}^{+\infty} \mathrm{u}_{\mathrm{n}}=\mathrm{u}(0)+\mathrm{u}^{\prime}(0) \mathrm{x}$

$+\mathrm{L}^{-1}\left[\sum_{\mathrm{n}=0}^{+\infty} \mathrm{a}_{\mathrm{n}} \mathrm{x}^{\mathrm{n}}\right]-\mathrm{pL}^{-1}\left[\sum_{\mathrm{n}=0}^{+\infty} \mathrm{a}_{\mathrm{n}} \mathrm{x}^{\mathrm{n}}\right]$

$-\mathrm{L}^{-1}\left[\mathrm{R}\left(\sum_{\mathrm{n}=0}^{+\infty} \mathrm{u}_{\mathrm{n}}\right)+\sum_{\mathrm{n}=0}^{+\infty} \mathrm{A}_{\mathrm{n}}\left(\mathrm{u}_{0}, \mathrm{u}_{1}, \ldots, \mathrm{u}_{\mathrm{n}}\right)\right]$,

where $\mathrm{p}$ is an artificial parameter and $\mathrm{a}_{\mathrm{i}}, \mathrm{i}=0,1, \ldots$ are unknown coefficients. We now define

$\mathrm{u}_{0}=\mathrm{u}(0)+\mathrm{u}^{\prime}(0) \mathrm{x}+\mathrm{L}^{-1}\left[\sum_{\mathrm{n}=0}^{+\infty} \mathrm{a}_{\mathrm{n}} \mathrm{x}^{\mathrm{n}}\right]=1+\frac{1}{2} \mathrm{a}_{0} \mathrm{x}^{2}$

$+\frac{1}{6} a_{1} x^{3}+\frac{1}{12} a_{2} x^{4}+\frac{1}{20} a_{3} x^{5}+\cdots$,

$\mathrm{u}_{1}=-\mathrm{pL}^{-1}\left[\sum_{\mathrm{n}=0}^{+\infty} \mathrm{a}_{\mathrm{n}} \mathrm{x}^{\mathrm{n}}\right]-\mathrm{L}^{-1}\left[\mathrm{R}\left(\mathrm{u}_{0}\right)+\mathrm{A}_{0}\left(\mathrm{u}_{0}\right)\right]$,

$\mathrm{u}_{2}=-\mathrm{L}^{-1}\left[\mathrm{R}\left(\mathrm{u}_{1}\right)+\mathrm{A}_{1}\left(\mathrm{u}_{0}, \mathrm{u}_{1}\right)\right]$,

To avoid calculation of $A_{n}, n=1,2, \ldots$, let determine $a_{i}, i=0,1, \ldots$ such that $\mathrm{u}_{1}=0$. Thus

$\left(-1-\frac{1}{2} \mathrm{a}_{0}-\frac{1}{2} \mathrm{pa}_{0}\right) \mathrm{x}^{2}+\left(-\frac{1}{12} \mathrm{a}_{1}-\frac{1}{6} \mathrm{pa}_{1}\right) \mathrm{x}^{3}$

$+\left(-\frac{1}{2} \mathrm{a}_{0}-\frac{1}{36} \mathrm{a}_{2}-\frac{1}{12} \mathrm{pa}_{2}\right) \mathrm{x}^{4}+\cdots=0$.

It can be easily shown that

$a_{0}=-\frac{2}{1+p}, \quad a_{1}=0, \quad a_{2}=\frac{36}{(1+p)(1+3 p)}, \quad a_{3}=0, \cdots$

Setting $\mathrm{p}=1$, results in 
$u(x)=1-\frac{1}{2} x^{2}+\frac{3}{8} x^{4}-\frac{5}{16} x^{6}+\cdots=\frac{1}{\sqrt{1+x^{2}}}$

which is the exact solution of the problem.

Example 3.2: Consider the inhomogeneous Emden-Fowler equation with $\alpha=2$ [1]

$u^{\prime \prime}+\frac{2}{x} u^{\prime}-\left(6+4 x^{2}\right) u=6-6 x^{2}-4 x^{4}$,

subject to the following initial conditions

$\mathrm{u}(0)=1, \quad \mathrm{u}^{\prime}(0)=0$.

Traditional ADM. Applying the traditional ADM yields

$u_{0}(x)=1+3 x^{2}-\frac{1}{2} x^{4}-\frac{2}{15} x^{6}$

$u_{1}(x)=-3 x^{2}+\frac{13}{6} x^{4}+\frac{53}{150} x^{6}-\frac{1}{20} x^{8}-\frac{4}{675} x^{10}$

$u_{2}(x)=6 x^{2}-\frac{53}{18} x^{4}-\frac{27}{250} x^{6}+\frac{869}{4200} x^{8}+\frac{1663}{121500} x^{10}$

$-\frac{53}{29700} x^{12}-\frac{8}{61425} x^{14}$

Consequently, the series solution obtained by the traditional ADM is as follows

$$
\begin{aligned}
& u(x)=1+6 x^{2}-\frac{23}{18} x^{4}+\frac{14}{125} x^{6}+\frac{659}{4200} x^{8} \\
& +\frac{943}{121500} x^{10}-\frac{53}{29700} x^{12}-\frac{8}{61425} x^{14}+\cdots
\end{aligned}
$$

Modified ADM. A similar procedure, described in previous example, leads to

$\mathrm{u}_{0}=\mathrm{u}(0)+\mathrm{u}^{\prime}(0) \mathrm{x}+\mathrm{L}^{-1}\left[\sum_{\mathrm{n}=0}^{+\infty} \mathrm{a}_{\mathrm{n}} \mathrm{x}^{\mathrm{n}}\right]=1+\frac{1}{2} \mathrm{a}_{0} \mathrm{x}^{2}$

$+\frac{1}{6} a_{1} x^{3}+\frac{1}{12} a_{2} x^{4}+\frac{1}{20} a_{3} x^{5}+\cdots$,

$\mathrm{u}_{1}=\mathrm{L}^{-1}\left[6-6 \mathrm{x}^{2}-4 \mathrm{x}^{4}\right]-\mathrm{pL}^{-1}\left[\sum_{\mathrm{n}=0}^{+\infty} \mathrm{a}_{\mathrm{n}} \mathrm{x}^{\mathrm{n}}\right]-\mathrm{L}^{-1}\left[\mathrm{R}\left(\mathrm{u}_{0}\right)\right]$,

where $L^{-1}=\int_{0}^{X} \int_{0}^{X}() d x d$.$x and R(u)=(2 / x) u^{\prime}-\left(6+4 x^{2}\right) u$. Now, by setting $\mathrm{u}_{1}(\mathrm{x})=0$, we find

$$
\begin{aligned}
& \left(6-\mathrm{a}_{0}-\frac{1}{2} \mathrm{pa}_{0}\right) \mathrm{x}^{2}+\left(-\frac{1}{6} \mathrm{a}_{1}-\frac{1}{6} \mathrm{pa}_{1}\right) \mathrm{x}^{3} \\
& +\left(-\frac{1}{6}+\frac{1}{4} \mathrm{a}_{0}-\frac{1}{18} \mathrm{a}_{2}-\frac{1}{12} \mathrm{pa}_{2}\right) \mathrm{x}^{4}+\cdots=0 .
\end{aligned}
$$

It can be shown that

$\mathrm{a}_{0}=\frac{12}{2+\mathrm{p}}, \mathrm{a}_{1}=0, \mathrm{a}_{2}=-\frac{6(\mathrm{p}-16)}{(2+\mathrm{p})(2+3 \mathrm{p})}, \mathrm{a}_{3}=0, \cdots$

Setting $\mathrm{p}=1$, yields $u(x)=1+2 x^{2}+\frac{1}{2} x^{4}+\frac{1}{6} x^{6}+\cdots=x^{2}$

$+\left(1+x^{2}+\frac{1}{2} x^{4}+\frac{1}{6} x^{6}+\cdots\right)$

which converges to the exact solution

$u(x)=x^{2}+e^{x^{2}}$

Example 3.3: Consider the inhomogeneous Emden-Fowler equation with $\alpha>2$ [1]

$u^{\prime \prime}+\frac{4}{x} u^{\prime}-\left(18 x+9 x^{4}\right) u=20-36 x^{3}-18 x^{6}$

with the following initial conditions

$\mathrm{u}(0)=1, \quad \mathrm{u}^{\prime}(0)=0$.

Traditional ADM. Using the traditional ADM leads to

$$
\begin{aligned}
& u_{0}(x)=1+10 x^{2}-\frac{9}{5} x^{5}-\frac{9}{28} x^{8}, \\
& u_{1}(x)=-40 x^{2}+3 x^{3}+\frac{54}{5} x^{5}+\frac{3}{10} x^{6} \\
& +\frac{297}{245} x^{8}-\frac{1539}{7700} x^{11}-\frac{81}{5096} x^{14}, \\
& u_{2}(x)=160 x^{2}-6 x^{3}-\frac{234}{5} x^{5}+\frac{39}{25} x^{6} \\
& -\frac{12519}{3430} x^{8}+\frac{9}{20} x^{9}+\frac{156573}{134750} x^{11}+\frac{9}{440} x^{12} \\
& +\frac{2873799}{63763700} x^{14}-\frac{365229}{47647600} x^{17}-\frac{729}{1936480} x^{20},
\end{aligned}
$$

Therefore, the series solution resulted from the traditional ADM is as follows

$$
\begin{aligned}
& u(x)=1+130 x^{2}-3 x^{3}-\frac{189}{5} x^{5}+\frac{93}{50} x^{6} \\
& -\frac{18927}{6860} x^{8}+\frac{9}{20} x^{9}+\frac{23571}{24500} x^{11}+\frac{9}{440} x^{12} \\
& +\frac{3720573}{127527400} x^{14}-\frac{365229}{47647600} x^{17}-\frac{729}{1936480} x^{20}+\cdots
\end{aligned}
$$

Modified ADM. In a manner similar to that described in previous examples, we can define

$$
\begin{aligned}
& u_{0}=u(0)+u^{\prime}(0) x+L^{-1}\left[\sum_{n=0}^{+\infty} a_{n} x^{n}\right]=1+\frac{1}{2} a_{0} x^{2} \\
& +\frac{1}{6} a_{1} x^{3}+\frac{1}{12} a_{2} x^{4}+\frac{1}{20} a_{3} x^{5}+\cdots \\
& u_{1}=L^{-1}\left[20-36 x^{3}-18 x^{6}\right]-p L^{-1}\left[\sum_{n=0}^{+\infty} a_{n} x^{n}\right]-L^{-1}\left[R\left(u_{0}\right)\right]
\end{aligned}
$$

where $L^{-1}=\int_{0}^{X} \int_{0}^{X}() d x d$.$x and R(u)=(4 / x) u^{\prime}-\left(18 x+9 x^{4}\right) u$. Now, if we set $\mathrm{u}_{1}(\mathrm{x})$ equal to zero, then we obtain 
$\left(10-2 \mathrm{a}_{0}-\frac{1}{2} \mathrm{pa}_{0}\right) \mathrm{x}^{2}+\left(3-\frac{1}{3} \mathrm{a}_{1}-\frac{1}{6} \mathrm{pa}_{1}\right) \mathrm{x}^{3}$

$+\left(-\frac{1}{9} \mathrm{a}_{2}-\frac{1}{12} \mathrm{pa}_{2}\right) \mathrm{x}^{4}+\cdots=0$.

It can be easily shown that

$a_{0}=\frac{20}{4+p}, \quad a_{1}=\frac{18}{2+p}, \quad a_{2}=0, \quad a_{3}=-\frac{36(p-1)}{(1+p)(4+p)}, \cdots$

Setting $\mathrm{p}=1$, results in

$u(x)=1+2 x^{2}+x^{3}+\frac{1}{2} x^{6}+\cdots=2 x^{2}$

$+\left(1+x^{3}+\frac{1}{2} x^{6}+\cdots\right)=2 x^{2}+e^{x^{3}}$,

which is the exact solution of the problem.

\section{Conclusion}

In this article, a new and effective modification of Adomian decomposition method was proposed to solve singular, EmdenFowler type, initial value problems with $\alpha=1,2$, and $>2$. As it was observed

- The method only requires the calculation of the first Adomian polynomial.

- The method provides the solution of the problems in the form of a convergent series, whereas the traditional ADM fails.

- The method overcomes the singularity at $\mathrm{x}=0$.

It is worth mentioning that the proposed method can be extended for solving systems of ordinary differential equations of EmdenFowler type as follows [23]

$\mathrm{u}_{1}^{\prime \prime}+\frac{\alpha_{1}}{\mathrm{x}} \mathrm{u}_{1}^{\prime}+\mathrm{f}_{1}\left(\mathrm{u}_{1}, \mathrm{u}_{2}\right)=\mathrm{g}_{1}(\mathrm{x}), \mathrm{u}_{1}(0)=\mathrm{a}_{1}, \mathrm{u}_{1}^{\prime}(0)=0$,

$u_{2}^{\prime \prime}+\frac{\alpha_{2}}{x} u_{2}^{\prime}+f_{2}\left(u_{1}, u_{2}\right)=g_{2}(x), u_{2}(0)=a_{2}, u_{2}^{\prime}(0)=0$.

\section{References}

[1] A.M. Wazwaz, A reliable treatment of singular Emden-Fowler initial value problems and boundary value problems, Applied Mathematics and Computation, 217 (2011), 10387-10395. http://dx.doi.org/10.1016/j.amc.2011.04.084.

[2] K. Parand, M. Dehghan, A.R. Rezaei, S.M. Ghaderi, An approximation algorithm for the solution of the nonlinear LaneEmden type equations arising in astrophysics using Hermite functions collocation method, Computer Physics Communications, $181 \quad$ (2010), 1096-1108 http://dx.doi.org/10.1016/j.cpc.2010.02.018.

[3] K. Parand, M. Shahini, M. Dehghan, Rational Legendre pseudospectral approach for solving nonlinear differential equations of Lane-Emden type, Journal of Computational Physics, 228 (2009), 8830-8840. http://dx.doi.org/10.1016/j.jcp.2009.08.029.

[4] A.M. Wazwaz, A new algorithm for solving differential equation of Lane-Emden type, Applied Mathematics and Computation, 118 (2001), 287-310. http://dx.doi.org/10.1016/S0096-3003(99)00223-

[5] S.J. Liao, A new analytic algorithm of Lane-Emden type equations, Applied Mathematics and Computation, 142 (2003), 1-16. http://dx.doi.org/10.1016/S0096-3003(02)00943-8

[6] O.P. Singh, R.K. Pandey, V.K. Singh, An analytic algorithm of Lane-Emden type equations arising in astrophysics using modified homotopy analysis method, Computer Physics Communications, 180

$1116-1124$ http://dx.doi.org/10.1016/j.cpc.2009.01.012.
[7] J.I. Ramos, Linearization techniques for singular initial-value problems of ordinary differential equations, Applied Mathematics and Computation, 161 (2005), 525-542. http://dx.doi.org/10.1016/j.amc.2003.12.047.

[8] A. Yildirim, T. Ozis, Solutions of singular IVPs of Lane-Emden type by homotopy perturbation method, Physics Letters A, 369 (2007), 70-76. http://dx.doi.org/10.1016/j.physleta.2007.04.072.

[9] X. Shang, P. Wu, X. Shao, An efficient method for solving EmdenFowler equations, Journal of the Franklin Institute, 346 (2009), 889-897. http://dx.doi.org/10.1016/j.jfranklin.2009.07.005.

[10] J.H. He, Variational approach to the Lane-Emden equation, Applied Mathematics and Computation, 143 (2003), 539-541. http://dx.doi.org/10.1016/S0096-3003(02)00382-X.

[11] J.I. Ramos, Series approach to the Lane-Emden equation and comparison with the homotopy perturbation method, Chaos, Solitons \& Fractals, $38 \quad$ (2008), 400-408. http://dx.doi.org/10.1016/j.chaos.2006.11.018.

[12] S.A. Yousefi, Legendre wavelets method for solving differential equations of Lane-Emden type, Applied Mathematics and Computation, 181 (2006), 1417-1422. http://dx.doi.org/10.1016/i.amc.2006.02.031.

[13] N.T. Shawagfeh, Nonperturbative approximate solution for LaneEmden equation, Journal of Mathematical Physics, 34 (1993), 4364-4369. http://dx.doi.org/10.1063/1.530005.

[14] A.S. Bataineh, M.S.M. Noorani, I. Hashim, Homotopy analysis method for singular IVPs of Emden-Fowler type, Communications in Nonlinear Science and Numerical Simulation, 14 (2009), 11211131. http://dx.doi.org/10.1016/j.cnsns.2008.02.004.

[15] M.S.H. Chowdhury, I. Hashim, Solutions of Emden-Fowler equations by homotopy perturbation method, Nonlinear Analysis: Real World Applications, 10 (2009), 104-115. http://dx.doi.org/10.1016/j.nonrwa.2007.08.017.

[16] H.R. Marzban, H.R. Tabrizidooz, M. Razzaghi, Hybrid functions for nonlinear initial-value problems with applications to LaneEmden type equations, Physics Letters A, 372 (2008), 5883-5886. http://dx.doi.org/10.1016/j.physleta.2008.07.055.

[17] M. Dehghan, F. Shakeri, Approximate solution of a differential equation arising in astrophysics using the variational iteration method, New Astronomy, 13 (2008), 53-59. http://dx.doi.org/10.1016/j.newast.2007.06.012.

[18] R.K. Pandey, N. Kumar, Solution of Lane-Emden type equations using Bernstein matrix of differentiation, New Astronomy, 17 (2012), 303-308. http://dx.doi.org/10.1016/j.newast.2011.09.005.

[19] B. Muatjetjeja, C.M. Khalique, Exact solutions of the generalized Lane-Emden equations of the first and second kind, Pramana Journal of Physics, 77 (2011), 545-554. http://dx.doi.org/10.1007/s12043-011-0174-4

[20] J.S. Duan, Convenient analytic recurrence algorithms for the Adomian polynomials, Applied Mathematics and Computation, 217 (2011), 6337-6348. http://dx.doi.org/10.1016/j.amc.2011.01.007.

[21] H. Aminikhah, J. Biazar, A new HPM for ordinary differential equations, Numerical Methods for Partial Differential Equations, 26 (2009), 480-489. http://dx.doi.org/10.1002/num.20413.

[22] K. Hosseini, J. Biazar, R. Ansari, P. Gholamin, A new algorithm for solving differential equations, Mathematical Methods in the $\begin{array}{llll}\text { Applied } & \text { Sciences, } & 35 & \text { (2012), }\end{array}$ http://dx.doi.org/10.1002/mma.1601.

[23] B. Muatjetjeja, C.M. Khalique, Lagrangian approach to a generalized coupled Lane-Emden system: Symmetries and first integrals, Communications in Nonlinear Science and Numerical Simulation, $\quad 15 \quad$ (2010), 1166-1171. http://dx.doi.org/10.1016/j.cnsns.2009.06.002. 